\title{
A bivariate matrix Padé-type method of the 2-D filters
}

\author{
Chuanqing $\mathrm{Gu}$ \\ Department of Mathematics, Shanghai University \\ Shanghai 200444, China \\ cqgu@staff.shu.edu.cn
}

\author{
Rongrong Cui \\ Department of Mathematics, Shanghai University \\ Shanghai 200444, China \\ cuirongrong943@sohu.com
}

\begin{abstract}
In this paper,according to the special generating polynomial, a class of bivariate matrix Padé-type approximation(BMPTA) is given by introducing a bivariate matrix-valued linear functional on the scalar polynomial space.An application in state-space realization of the 2-D filters is also given in the end.
\end{abstract}

Keywords-matrix bivariate Padé-type approximation, linear functional, 2-D filters

\section{INTRODUCTION}

The problem of matrix padé approximants and the wide application has been studied for a long time in many fields, such as in scattering physics, multiport network synthesis, modelreduction, design of multi-input multi-output digital filters[1,2].Bose and Basu(3)introduced the classic bivariate matrix valued padé approximant and discussed the existence, nonuniqueness and recursive computation with inverse matrix. Author Gu[4]defined generalized inverse Thieletype bivariate matrix padé approximant(BGMPA) with scalar denominator polynomials. As compared the computation to classic Padé approximant, BGMPA does not need to define left-handed and right-handed approximants and it is easy and effective. However, BGMPA has its restriction, that is, the degree of the denominator polynomial is always even because of the divisibility of BGMPA.

Following the Padé-type approximants[5], Gu[6] gave a matrix-valued linear functional on the scalar polynomial space and defined the 1-D matrix Padé-type approximant. Tao and $\mathrm{Gu}$ [7]gave a general 2-D matrix padé-type approxinmation in the inner product space(BMPTA).In this paper we give a special class of BMPTA by giving a scalar generating polynomial.

\section{DEFINITION OF BMPTA}

Let $\hat{P}$ be the set of scalar polynomials in two real variable whose coefficients belong to the complex field C. Consider a bivariate matrix-valued function $\mathrm{f}(\mathrm{x}, \mathrm{y})$ has formal series in two variable $\mathrm{x}$ and $\mathrm{y}$

$$
\begin{aligned}
f(x, y)= & \sum_{i=0}^{\infty} \sum_{j=0}^{\infty} c_{i j} x^{i} y^{j}=c_{00}+\left(c_{10} x+c_{01} y\right)+\left(c_{20} x^{2}+c_{11} x y+c_{02} y^{2}\right) \\
& +\left(c_{30} x^{3}+c_{21} x^{2} y+c_{12} x y^{2}+c_{03} y^{3}\right)+\cdots
\end{aligned}
$$

where $C_{i j} \in C^{M \times N}$.

Let $\hat{\varphi}: \hat{P} \rightarrow C^{M \times N}$ be a generalized linear function on $\hat{P}$,acting on s, t, defined by

$$
\hat{\varphi}\left(s^{i} t^{j}\right)=c_{i j}, \quad i, j=0,1, \cdots
$$

Suppose that $|\mathrm{tx}|<1$, $\mid$ sy $\mid<1$, we have

$(1-t x)^{-1}=1+t x+(t x)^{2}+\cdots,(1-s y)^{-1}=1+s y+(s y)^{2}+\cdots$

For the given series(1),from (2)and (3) we get $\hat{\varphi}(1-t x)^{-1}(1-s y)^{-1}=\hat{\varphi}\left(1+t x+(t x)^{2}+\cdot\right)\left(1+s y+(s y)^{2}+\cdot\right)=\hat{\varphi}\left(1+(t x+s y)\left[(t x)^{2}+(s t) x y\right.\right.$ $\left.\left.+(s y)^{2}\right]+\left[(t x)^{3}+(t x)^{2}(s y)+(t x)(s y)^{2}+(s y)^{3}\right]+\cdot \cdot\right)=c_{00}+\left(c_{10} x+c_{01} y\right)+\left(c_{00} x^{2}+c_{11} x y+c_{02} y^{2}\right)$ $+\left(c_{30} x^{3}+c_{21} x^{2} y+c_{12} x y^{2}+c_{03} y^{3}\right)+\cdot=f(x, y)$

Let $\mathrm{V}(\mathrm{x}, \mathrm{y})$ be a bivariate scalar polynomial:

$$
V(x, y)=\sum_{i=0}^{n_{1}} \sum_{j=0}^{n_{2}} b_{i j} x^{i} y^{j}
$$

And assume $b_{n_{1}, n_{2}} \neq 0$. Define the bivariate matrixvalued polynomial $\mathrm{W}(\mathrm{x}, \mathrm{y})$ :

$$
W(x, y)=\hat{\varphi}\left(\frac{V(x, y)+V(t, s)-V(t, y)-V(x, s)}{(t-x)(s-y)}\right)
$$

Where $\hat{\varphi}$ acts on $\mathrm{t}$ and s,and $\mathrm{x}, \mathrm{y}$ are parameters. Let

$$
\begin{aligned}
& \tilde{V}(x, y)=x^{n_{1}} y^{n_{2}} V\left(x^{-1}, y^{-1}\right) \\
& \tilde{W}(x, y)=x^{n_{1}-1} y^{n_{2}-1} W\left(x^{-1}, y^{-1}\right)
\end{aligned}
$$

Definition 2.1 Let $\tilde{V}(x, y) \neq 0, f(x, y)$ be a matrix power series, then the matrix-valued rational function $R_{n_{1}, n_{2}}(x, y)=\frac{\tilde{W}(x, y)}{\tilde{V}(x, y)}$ is defined to be a bivariate matrix padé-type approximation(BMPTA) of degree $\left(n_{1}-1, n_{2}-1 / n_{1}, n_{2}\right)$, which satisfies the approximational condition:

$$
\begin{aligned}
& \tilde{V}(x, y) f(x, y)-\tilde{W}(x, y) \\
& =\sum_{i=n_{1}}^{\infty} d_{i 0} x^{i}+\sum_{j=n_{2}}^{\infty} d_{0 j} y^{j}+\sum_{i=n_{1}}^{\infty} \sum_{j=1}^{\infty} d_{i j} x^{i} y^{j}+\sum_{i=1}^{\infty} \sum_{j=n_{2}}^{\infty} d_{i j} x^{i} y^{j}
\end{aligned}
$$

where all the coefficients $d_{i j}$ are $M \times N$ matrices of constants, and $V(x, y)$ is said to be the generating polynomial.

We denote a BMPTA of degree $\left(n_{1}-1, n_{2}-1 / n_{1}, n_{2}\right)$

by $\left(n_{1}-1, n_{2}-1 / n_{1}, n_{2}\right)_{f}(x, y)$ 
Remark 2.2 In fact,the bivariate matrix power series in (1) can be expanded by the following form: $f(x, y)=\sum_{i=0}^{\infty} c_{i 0} x^{i}+\sum_{j=0}^{\infty} c_{0 j} y^{j}+\sum_{i=1}^{\infty} \sum_{j=1}^{\infty} c_{i j} x^{i} y^{j}$

and the approximation condition in definition 2.1 indicates that $\left(n_{1}-1, n_{2}-1 / n_{1}, n_{2}\right)_{f}(x, y)=R_{n_{1}, n_{2}}(x, y)$ is to agree with (9) in all $n_{1} n_{2}=n_{1}+\left(n_{2}-1\right)+m$ terms. These

$n_{1} n_{2}$ terms in (9)include

(i) $n_{1}$ terms before $\sum_{i=n_{1}}^{\infty} d_{i 0} x^{i}: c_{00}, c_{10} x, \cdots, c_{n_{1}-1,0} x^{n_{1}-1}$

$$
n_{2}-1
$$

terms

before $\sum_{j=n_{2}}^{\infty} d_{0 j} y^{j}: c_{01} y, \cdots, c_{0, n_{2}-1} y^{n_{2}-1}$

(iii) $m=n_{1} n_{2}-\left(n_{1}+n_{2}\right)+1$ terms before

$$
\sum_{i, j}^{\infty} d_{i j} x^{i} y^{j}: c_{11} x y, c_{21} x^{2} y, c_{12} x y^{2}, \cdots, c_{n_{1}-1, n_{2}-1} x^{n_{1}-1} y^{n_{2}-1}
$$

Example2.3 Let $n_{1}=n_{2}=3$, then $(2,2 / 3,3)_{f}(x, y)$

Is to agree with (9) in $n_{1} n_{2}=9$ terms:

(i) $n_{1}=3$ terms : $c_{00}, c_{10} x, c_{20} x^{2}$

(ii) $n_{2}-1=2$ terms : $c_{01} y, c_{02} y^{2}$

(iii) $m=n_{1} n_{2}-\left(n_{1}+n_{2}\right)+1=4$ terms :

$$
c_{11} x y, c_{21} x^{2} y, c_{12} x y^{2}, c_{22} x^{2} y^{2}
$$

III. A CONSTRUCTION OF BMPTA

Now we restrict the generating polynomial $V_{n_{1}, n_{2}}(x, y)=u_{n_{1}}(x) v_{n_{2}}(y)$ Where

$u_{n_{1}}(x)=\left(x-t_{1}\right)\left(x-t_{2}\right) \cdots\left(x-t_{n_{1}}\right), v_{n_{2}}(y)=\left(y-s_{1}\right)\left(y-s_{2}\right) \cdots\left(y-s_{n_{2}}\right)$

Obviously, the zeros of $u_{n_{1}}:\left\{t_{i}: i=1,2, \cdots, n_{1}\right\}$, the zeros of $v_{n_{1}}:\left\{s_{j}: j=1,2, \cdots, n_{2}\right\}$

From (6)(7)(8)we obtain

$\frac{\tilde{W}(x, y)}{\tilde{V}(x, y)}=\hat{\varphi}\left(\frac{x^{n_{1}-1}}{\tilde{u}_{n_{1}}(x)} \frac{u_{n_{1}}\left(x^{-1}\right)-u_{n_{1}}(t)}{x^{-1}-t} \frac{y^{n_{2}-1}}{\tilde{v}_{n_{2}}(y)} \frac{v_{n_{2}}\left(y^{-1}\right)-v_{n_{2}}(s)}{y^{-1}-s}\right)$

Where

$\tilde{u}_{n_{1}}(x)=\prod_{i=1}^{n_{1}}\left(1-t_{i} x\right), \tilde{v}_{n_{2}}(y)=\prod_{j=1}^{n_{2}}\left(1-s_{j} y\right)$

From (10), we define

$$
\begin{aligned}
T_{n_{1}}(x) & =\frac{x^{n_{1}-1}\left(u_{n_{1}}\left(x^{-1}\right)-u_{n_{1}}(t)\right)}{x^{-1}-t} \\
& =a_{0}(x)+a_{1}(x) t+\cdots+a_{n_{1}-1}(x) t^{n_{1}-1}
\end{aligned}
$$

Where $a_{i}(x)$ is the polynomial with respect to $x$, $b_{j}(y)$ is the polynomial with respect to $y$.

Let $A=\left(a_{i j}\right), B=\left(b_{i j}\right) \in C^{M \times N}$, in [10] the matrix direct inner product is defined by $(A, B)=\sum_{i=1}^{M} \sum_{j=1}^{N} a_{i j} b_{i j}$

$T_{n_{1}}(x) T_{n_{2}}(y)$ can be written as follows:

$T_{n_{1}}(x) T_{n_{2}}(y)$

$=a_{0}(x) b_{0}(y)+a_{0}(x) b_{1}(y) s+\cdots+a_{0}(x) b_{n_{2}-1}(y) s^{n_{2}-1}+a_{1}(x) b_{0}(y) t$

$+a_{1}(x) b_{1}(y) t s+\cdots+a_{1}(x) b_{n_{2}-1}(y) t s^{n_{2}-1}+\cdots+a_{n_{1}-1}(x) b_{0}(y) t^{n_{1}-1}$

$+a_{n_{1}-1}(x) b_{1}(y) t^{n_{1}-1} s+\cdots+a_{n_{1}-1}(x) b_{n_{2}-1}(y) t^{n_{1}-1} s^{n_{2}-1}$

$=<\left[t^{i-1} s^{j-1}\right],\left[a_{i-1}(x) b_{j-1}(y)\right]>$

Where $\left[t^{i-1} s^{j-1}\right]=\left(\begin{array}{cccc}1 & s & \cdots & s^{n_{2}-1} \\ t & t s & \cdots & t s^{n_{2}-1} \\ \cdots & \cdots & \cdots & \cdots \\ t^{n_{1}-1} & t^{n_{1}-1} s & \cdots & t^{n_{1}-1} s^{n_{2}-1}\end{array}\right)$,

$\left[a_{i-1}(x) b_{j-1}(y)\right]=\left(\begin{array}{cccc}a_{0}(x) b_{0}(y) & a_{0}(x) b_{1}(y) & \cdots & a_{0}(x) b_{n_{2}-1}(y) \\ a_{1}(x) b_{0}(y) & a_{1}(x) b_{1}(y) & \cdots & a_{1}(x) b_{n_{2}-1}(y) \\ \cdots & \cdots & \cdots & \cdots \\ a_{n_{1}-1}(x) b_{0}(y) & a_{n_{1}-1}(x) b_{1}(y) & \cdots & a_{n_{1}-1}(x) b_{n_{2}-1}(y)\end{array}\right)$

Then $\hat{\varphi}\left(T_{n_{1}}(x) T_{n_{2}}(y)\right)=\hat{\varphi}<\left[t^{i-1} s^{j-1}\right]$,

$\left[a_{i-1}(x) b_{j-1}(y)\right]>=<H_{n_{1}, n_{2}}, G_{n_{1}, n_{2}}>$, where

$H_{n_{1}, n_{2}}=\left(\begin{array}{cccc}c_{00} & c_{01} & \cdots & c_{0, n_{2}-1} \\ c_{10} & c_{11} & \cdots & c_{1, n_{2}-1} \\ \cdots & \cdots & \cdots & \cdots \\ c_{n_{1}-1,0} & c_{n_{1}-1,1} & \cdots & c_{n_{1}-1, n_{2}-1}\end{array}\right)$,

$G_{n_{1}, n_{2}}=\left[a_{i-1}(x) b_{j-1}(y)\right]$

According to the above analysis, we can easily get the following theorem:

Theorem3.1 Let $\tilde{V}(0,0) \neq 0$, then

$\left(n_{1}-1, n_{2}-1 / n_{1}, n_{2}\right)_{f}(x, y)=\frac{1}{\tilde{u}_{n_{1}}(x) \tilde{v}_{n_{2}}(y)}<H_{n_{1}, n_{2}}, G_{n_{1}, n_{2}}>$

Remark: $H_{n_{1}, n_{2}}$ in (11) only contains the coefficients of $f(x, y)$, and $G_{n_{1}, n_{2}}$ in (11) only contains the zeros of. $V_{n_{1}, n_{2}}(x, y)=u_{n_{1}}(x) v_{n_{2}}(y)$

IV. A PARTIAL REALIZATION OF THE 2-D FILTERS

The realization problem of two-dimensional linear filters is an important problem of model reduction in control 
theory. The input-output behavior of such a system is defined by formal power series in two-variables.The authors of[9] proved that if the power series is rational, the dynamics of the F-M linear filter is described by updating equations on finite-dimensional local state space.

Let $\mathrm{K}$ be the complex plane $\mathrm{C}$ or real plane $\mathrm{R}$. Let the state equation of 2-D linear discrete time invariable system for Fornasin-Marchesini model(F-M system) in [9] be $\sum=\left(A_{0}, A_{1}, A_{2}, B, C\right)$ :

$X(h+1, k+1)=A_{0} X(h, k)+A X(h+1, k)+A_{2} X(h, k+1)+B U(h, k)$

$Y(h, k)=C K(h, k)$.

Where $X(h, k) \in K^{q}$ is the local state vector, $U(h, k)$ is the input vector, $Y(h, k)$ is the output vector,

$A_{0}, A_{1}, A_{2} \in K^{q \times l}$.Boundary conditions for (12) are given by $X(h, 0), X(0, k), h, k=0,1,2 \cdots$ By acting bivariate Z-transfom on both sides of (12) we obtain the transfer function of F-M system

$$
G=G(x, y)=C\left(I-A_{0} x y-A_{1} x-A_{2} y\right)^{-1} B
$$

Assume that the matrix-valued polynomial

$\left(I-A_{0} x y-A_{1} x-A_{2} y\right)^{-1}$ have the inverse matrix given by

$$
f(x, y)=\left(I-A_{0} x y-A_{1} x-A_{2} y\right)^{-1}=\sum_{i, j=0}^{\infty} c_{i j} x^{i} y^{j}
$$

Where $c_{i j} \in K^{q \times q}$.Thus the output vector $\mathrm{Y}$ can be written as

$$
Y(x, y)=C \sum_{i, j=0}^{\infty} X(h, k) x^{i} y^{j}=C f(x, y) B U
$$

\section{Definition 4.1 Let}

$$
\left(n_{1}-1, n_{2}-1 / n_{1}, n_{2}\right)_{f}(x, y)=\tilde{W}(x, y) / \tilde{v}(x, y)
$$

be a BMPTA for $f(x, y)$ in (14). Replace $f(x, y)$ by $\left(n_{1}-1, n_{2}-1 / n_{1}, n_{2}\right)_{f}(x, y)$ in (13)(15) respectively, such that

$$
\begin{aligned}
& G_{n_{1}, n_{2}}(x, y)=C\left(n_{1}-1, n_{2}-1 / n_{1}, n_{2}\right)_{f}(x, y) B, \\
& Y_{n_{1}, n_{2}}(x, y)=C\left(n_{1}-1, n_{2}-1 / n_{1}, n_{2}\right)_{f}(x, y) B U
\end{aligned}
$$

Thus

$$
\sum_{n_{1}, n_{2}}=\left(A_{0}, A_{1}, A_{2}, B, C ;\left(n_{1}-1, n_{2}-1 / n_{1}, n_{2}\right)_{f}(x, y)\right)
$$

is called a 2-D partial realization of type

$\left(n_{1}-1, n_{2}-1 / n_{1}, n_{2}\right)$ for F-M system.

Example4.2[8] Consider the following bivariate matrix power series:

$$
f(x, y)=\left(I-A_{0} x y-A_{1} x-A_{2} y\right)^{-1}=\sum_{i, j=0}^{\infty} c_{i j} x^{i} y^{j}
$$

$$
\begin{aligned}
A_{0}=0, A_{1}=\left(\begin{array}{llll}
0 & 0 & 0 & 0 \\
0 & 0 & 0 & 0 \\
1 & 0 & 2 & 0 \\
0 & 1 & 1 & 1
\end{array}\right), \\
A_{2}=\left(\begin{array}{cccc}
1 & 2 & 0 & -1 \\
-1 & 0 & 1 & 0 \\
0 & 0 & 0 & 0 \\
0 & 0 & 0 & 0
\end{array}\right), \\
B=\left(\begin{array}{cccc}
1 & 1 & 1 & 0 \\
-1 & 0 & 2 & 1
\end{array}\right)^{T}, C=\left(\begin{array}{cccc}
1 & 0 & 0 & 1 \\
1 & -1 & 1 & -1
\end{array}\right)
\end{aligned}
$$

Step1 Compute

$$
\begin{aligned}
& \tilde{V}(x, y)=\operatorname{det}\left(I-A_{0} x y-A_{1} x-A_{2} y\right) \\
& =9 x^{2} y^{2}-x^{2} y-9 x y^{2}+2 x^{2}+2 y^{2}+3 x y-3 x-y+1
\end{aligned}
$$

Let $n_{1}=n_{2}=2$, and let

$$
\begin{aligned}
& V(x, y)=x^{2} y^{2} \tilde{V}\left(x^{-1}, y^{-1}\right) \\
& \text { Step2 Compute } \\
& W(x, y)=\hat{\varphi}(t s+t y+x s+x y-t-x-3 s-3 y+3) \\
& =c_{00} x y+\left(c_{01}-c_{00}\right) x+\left(c_{10}-3 c_{00}\right) y+\left(c_{11}+3 c_{00}-c_{10}-3 c_{01}\right) \\
& \quad \text { let } \tilde{W}=x^{n_{1}-1} y^{n_{2}-1} W\left(x^{-1}, y^{-1}\right) \\
& \text { Step3 Compute } \\
& G_{2,2}(x, y)=C(1,1 / 2,2)_{f}(x, y) B=\frac{1}{\tilde{V}(x, y)} C \tilde{W B} \\
& =\frac{1}{\tilde{V}(x, y)}\left(\begin{array}{cc}
-10 x y-2 x+2 y+1 & 3 x y+3 x-2 y \\
-9 x y-2 x+2 y+1 & 4 x y-5 y
\end{array}\right)
\end{aligned}
$$

We can verify that $(1,1 / 2,2)_{f}(x, y)$ is to agree with (12) in $n_{1} n_{2}=4$ terms: $c_{00}, c_{10} x, c_{01} y, c_{11} x y$. From definition $4.1, \quad \sum_{2,2}=\left(A_{0}, A_{1}, A_{2,} B, C ;(1,1 / 2,2)_{f}\right)$ is the partial realization of type $(1,1 / 2,2)$ and

$$
Y_{2,2}(x, y)=C(1,1 / 2,2)_{f} B U
$$

\section{REFERENCES}

[1] Baker,G.A. and Graves-Morris,P.R., "Padé Approximation ,Part II” , Addison-Wesley Publishing Company,1981.

[2] J.R. Cardoso, F.S. Leite, "Padé and Gregory error estimates for the logarithm of block triangular matrices”, Appl. Numer. Math., 56(2006) 253-267.

[3] N. K. Bose and S. Basu, "Theory and recursive computation of 1-D matrix Padé approximants”, IEEE Trans. Circuits Syst., CAS-27(1980)323325.

[4] Chuan-qing Gu, "A Practical Two-Dimensional Thiele-Type Matrix Padé Approximation”, IEEE Transactions on Automatic Control,48(2003), 2259-2263.

[5] C.Brezinski, "Padé-type Approximation and General Orthogonal Polynomials”,in:ISNM, 50Birkha user,Basel, 1980. 
Proceedings of the 2012 2nd International Conference on Computer and Information Application (ICCIA 2012)

[6] Chuan-qing Gu,“Matrix Padé-type approximant and directional matrix Padé approximant in the inner product space”,J. Comput. Appl. Math., 164165(2004),365-385.
[7] Youtian Tao, Chuanqing Gu, "A two-dimensional matrix Padé-type approximation in the inner product space“, J. Comput. Appl. Math., 231(2009), 680-695.

[8] C. Gu“,Matrix valued rational approxinmation and applications in control theory”,Shanghai University Doctor Thesis,2004 